\title{
CircRNA hsa_circ_0030018 regulates the development of glioma via regulating the miR-1297/RAB21 axis
}

\author{
Dewen SONG, Lin YE, Zengliang XU, Yanfei JIN, Lintao ZHANG* \\ Department of Neurosurgery, Jiaozhou People's Hospital, Qingdao, Shandong, China \\ ${ }^{*}$ Correspondence: $z$ tt93899@163.com
}

Received July 02, 2020 / Accepted November 30, 2020

\begin{abstract}
Circular RNAs (circRNAs) play a crucial role in tumor occurrence and progression. And the dysregulated circRNAs are reported to be relevant to glioma development. Nevertheless, the function and regulatory mechanism of hsa_circ_0030018 in glioma progression are largely indistinct. The abundances of hsa_circ_0030018, miR-1297, and RAB21 were detected using quantitative real-time polymerase chain reaction or western blot. Cell proliferation was assessed via colony formation assay and 3-(4,5-dimethyl-2-thiazolyl)-2,5-diphenyl-2-H-tetrazolium bromide (MTT) assay. Cell apoptosis and cell cycle progression were evaluated by flow cytometry. Cell migration and invasion were examined using transwell assay and wound healing assay. The protein levels were measured by western blot. The interaction between miR-1297 and hsa_circ_0030018 or RAB21 was validated via dual-luciferase reporter analysis, RNA immunoprecipitation (RIP), and RNA pull-down assays. A xenograft model experiment was performed to analyze the function of hsa_circ_0030018 on tumor growth in vivo. hsa circ_0030018 and RAB21 levels were enhanced, and the miR-1297 level was reduced in glioma tissues and cells. The silence of hsa_circ_0030018 or overexpression of miR-1297 impeded cell proliferation, metastasis, and expedited cell apoptosis and cycle arrest in glioma cells. Furthermore, hsa_circ_0030018 modulated glioma malignant behaviors via sponging miR-1297, and miR-1297 suppressed glioma development via targeting RAB21. Moreover, hsa_circ_0030018 knockdown inhibited tumor growth in vivo. The hsa_circ_0030018 knockdown repressed glioma progression by mediating the miR-1297/RAB21 pathway, providing potential therapeutic targets for glioma treatment.
\end{abstract}

Key words: glioma, hsa_circ_0030018, miR-1297, RAB21

Glioma is a common malignant brain tumor, which is a serious threat to human health $[1,2]$. Because of the high postoperative recurrence rate and resistance to chemotherapy, the outcomes of glioma patients remain poor and it is a challenge to completely cure the patients with glioma [3, 4]. Despite numerous improvements in understanding the pathogenesis of glioma, the molecular mechanisms of glioma genesis are still mainly undiscovered. In consequence, it is urgent to explore new therapeutic targets for glioma treatment and elucidate the latent mechanism of the glioma process.

Circular RNAs (circRNAs) are a family of non-coding RNAs (ncRNAs) harboring covalently closed loops, and exert pivotal roles in specific diseases via acting as microRNA (miRNA) sponges $[5,6]$. Lots of evidence have authenticated that abnormally expressed circRNAs are closely related to the tumorigenesis and development of glioma $[7,8]$. For instance, circSMO742 could expedite glioma growth by targeting miR-338-3p to enhance SMO expres- sion [9]. Silencing circNFIX impeded glioma progression via the miR-378e/RPN2 axis [10]. hsa_circ_0030018 (also known as circ-POSTN) has been reported to serve as a decoy of miR-599 to prevent esophageal carcinoma progression by regulating ENAH expression [11]. Nevertheless, the biological action of hsa_circ_0030018 in glioma has not been perfectly clarified yet. Exceptionally, one research reported that hsa_circ_0030018 had been verified to accelerate glioma cell growth and invasion via miR-1205 [12]. While other regulatory pathways of hsa_circ_0030018 in glioma were not clear. Therefore, it is indispensable to explore the precise action and mechanism of hsa_circ_0030018 in the carcinogenesis of glioma.

microRNAs (miRNAs) are a kind of pivotal regulators of genetic expression, which can regulate gene expression at the post-transcriptional level in both physiological and pathological conditions $[13,14]$. Mounting studies have unveiled that miR-1297 level was decreased and the overexpression of miR-1297 could impede cell development via regulating 
different downstream genes in diverse cancers, such as pancreatic cancer [15], gastric cancer [16], prostate cancer [17], and so on. In addition, the miR-1297 abundance was visibly lessened and miR-1297 overexpression could hinder tumorigenesis in glioma via functional target HMGA1 [18]. Rab GTPase 21 (RAB21) is a member of the Rab GTPase family [19]. RAB21 deficiency might restrain proliferation and facilitate apoptosis in human glioma cells [20], which hinted that RAB21 might perform a carcinogenic role in glioma. Even so, whether miR-1297 and RAB21 participate in the modulatory action of hsa_circ_0030018 in glioma development is vague.

In this research, we first inspected hsa_circ_0030018 abundance in glioma tissues and cell lines and revealed its role in cancer development in vitro and in vivo. Furthermore, we explored the latent molecular mechanism of the hsa_circ_0030018/miR-1297/RAB21 axis in glioma cells through gain- and loss-of-function experiments, which provided a fresh theoretical basis and potential targets for glioma therapy.

\section{Patients and methods}

Clinical tissues and ethics statement. 40 tumor samples from glioma patients and 40 normal brain tissues from patients who received surgery because of other reasons except glioma were obtained from Jiaozhou People's Hospital and stored at $-80^{\circ} \mathrm{C}$ for further subsequent research. All participants did not receive another radiotherapy, chemotherapy, or related therapies before study initiation. Meanwhile, all participants were completely informed about this research purpose and signed written informed consent before sample collection. This research was permitted by the Ethics Committee of Jiaozhou People's Hospital.

Cell culture. Human glioma cell lines (A172, U251, LN229, and T98) and normal human astrocytes (NHAs) were provided by the Cell Bank of the Chinese Academy of Sciences (Shanghai, China). All glioma cell lines were grown in Dulbecco's modified Eagle's medium (DMEM; Invitrogen, Carlsbad, CA, USA) plus $10 \%$ fetal bovine serum (FBS; Hyclone, Logan, UT, USA) and 1\% antibiotics (Invitrogen). NHAs were cultured with astrocyte medium (Gibco, Grand Island, NY, USA). All the cells were cultivated in a $5 \% \mathrm{CO}_{2}$ humidified air atmosphere at $37^{\circ} \mathrm{C}$.

Cell transfection. For circ_0030018 silence, small-interfering RNAs (siRNAs) against hsa_circ_0030018 (si-hsa_ circ_0030018\#1, \#2, and \#3) or short hairpin RNAs (shRNAs) targeting hsa_circ_0030018 (sh-hsa_circ_0030018) were transfected into U251 and LN229 cells, and a non-target siRNA (si-NC) or shRNA (sh-NC) was employed as the negative control, respectively. For miR-1297 alteration, the synthetic miR-1297 mimics or miR-1297 inhibitor were introduced into U251 and LN229 cells, with a corresponding scrambled sequence (NC mimics or NC inhibitor) as the negative control. hsa_circ_0030018 or RAB21 upregulation was performed using hsa_circ_0030018 or RAB21 overexpression plasmid, with a non-target pCD-ciR or pcDNA3.1 ( $\mathrm{pcDNA}$ ) vector as a negative control. All oligonucleotides and plasmids were bought from Origene (Beijing, China) and transfected into U251 and LN229 cells for 48 h using Lipofectamine 3000 (Invitrogen) based on the specification when cell confluence reached $\sim 80 \%$. The transfection efficacy was detected via qRT-PCR or western blot at $24 \mathrm{~h}$ post-transfection.

Quantitative real-time polymerase chain reaction (qRT-PCR). Total RNA was isolated using TransZol(Transgen Biotech, Beijing, China), and reversely transcribed into cDNA using PrimeScript RT Reagent kit (Takara, Dalian, China) or microRNA Reverse Transcription Kit (Qiagen, Hilden, Germany). Finally, the cDNA, SYBR Green (Solarbio, Beijing, China), and specific primers (Sangon, Shanghai, China) were employed to perform qRT-PCR on the PCR system. The relative transcript expression of specific genes was evaluated based on the $2^{-\Delta \Delta \mathrm{Ct}}$ method and normalized to glyceraldehyde-3-phosphate dehydrogenase (GAPDH) or small nuclear RNA U6. The primers of the relative genes included: hsa_circ_0030018 (sense, 5'-ATAAACTCCTCTATCCAGCA-3'; antisense, TCCACAGGCACTCCATCAAT-3'), POSTN (sense, 5'-GAGACACACCCGTGAGGAAG-3'; antisense, 5'-ACCACAGGAGGCTAACTCCA-3'), miR-1297 (sense, 5'-TCGGCAGGTTCAAGTAATT-3'; antisense, 5'-CTCAACTGGTGTCGTGGA-3'), RAB21 (sense, 5'-GTCTCGCTCTGTCACCC-3'; antisense, 5'-CACGCCTGTAATCCC-3'), U6 (sense, 5'-AATTGGAACGATACAGAGAAGATTAGC-3'; antisense, 5'-TATGGAACGCTTCACGAATTTG-3'), and GAPDH (sense, 5'-GAATGGGCAGCCGTTAGGAA-3'; antisense, 5'-AAAAGCATCACCCGGAGGAG-3’).

Ribonuclease R (RNAse R) and Actinomycin D treatment. For RNase R digestion test, total RNA obtained from U251 and LN229 cells was incubated with $3 \mathrm{U} / \mu \mathrm{g}$ RNAse R or not (GeneSeed, Guangzhou, China) for $15 \mathrm{~min}$ at $37^{\circ} \mathrm{C}$. Additionally, U251 and LN229 cells were stimulated with $2 \mu \mathrm{g} / \mathrm{ml}$ Actinomycin D (Solarbio). Then, the expression of hsa_circ_0030018 or linear POSTN mRNA was examined using qRT-PCR.

Subcellular fractionation assay. The subcellular localization of hsa_circ_0030018 was measured by Cytoplasmic and Nuclear RNA Purification Kit (Norgen Biotek Corp., Belmont, MA, USA). The nuclear RNA and cytoplasmic RNA were obtained from U251 and LN229 cells using the above kit in compliance with the recommendations. Finally, the proportion of hsa_circ_0030018 in cytoplasmic and nucleus fractions was determined using qRT-PCR. GAPDH or U6 was used as the reference of cytoplasmic RNA or nuclear RNA, respectively.

3-(4,5-dimethylthiazol-2-yl)-2,5-diphenyltetrazolium bromide (MTT) assay and colony formation assay. MTT assay and colony formation assay were employed for quantification analysis of cell proliferation. For MTT assay, 
U251 and LN229 cells $\left(2 \times 10^{3}\right.$ cells/well) were seeded into 96-well plates and incubated for $1,2,3$, or 4 days at $37^{\circ} \mathrm{C}$. At each time point, MTT ( $5 \mathrm{mg} / \mathrm{ml}$; Solarbio) was added to incubate cells for an additional $4 \mathrm{~h}$. Subsequently, $100 \mu \mathrm{l}$ dimethyl sulfoxide (Solarbio) was added to each well. Then the optical density (OD) value at $490 \mathrm{~nm}$ was determined by a microplate reader (Bio-Gene Technology, Guangzhou, China). For colony formation assay, U251 and LN229 cells (500 cells/well) were plated into 6-well plates and cultivated for 14 days. Next, the cells were fixed with $4 \%$ paraformaldehyde (Solarbio) and dyed with $0.5 \%$ crystal violet (Solarbio). The colony formation number was counted by a microscope (Nikon, Tokyo, Japan).

Cell apoptosis and cell cycle assay. Cell apoptosis assay was performed by using Annexin V-fluorescein isothiocyanate (FITC) apoptosis detection kit (BD Pharmingen, San Diego, CA, USA). Briefly, at 48 h post-transfection, U251 and LN229 cells were collected and washed. Then, cells were double-stained with $5 \mu$ l of Annexin V labeled with FITC and $5 \mu$ l of propidium iodide (PI) in the dark for $30 \mathrm{~min}$ at $4{ }^{\circ} \mathrm{C}$. Apoptotic cells were detected by a FACScan flow cytometer (Becton Dickinson, Mountain View, CA, USA).

For cell cycle detection, $100 \mu \mathrm{l}$ of cell suspension was treated with $10 \mu \mathrm{M}$ RNAse $\mathrm{R}$ to remove RNA, then DNA was marked with $20 \mathrm{mg} / \mathrm{ml}$ PI staining solution (Solarbio) for $20 \mathrm{~min}$ at $37^{\circ} \mathrm{C}$. Cell cycle distribution at different phases was assessed using a FACScan flow cytometer (Becton Dickinson).

Transwell assay. Cell migration and invasion were detected through transwell chambers (Corning Inc., Corning, NY, USA). The procedures of migration and invasion were similar, but the upper compartment of the chambers was pre-coated with Matrigel (BD Bioscience, San Jose, CA, USA) for invasion assay. U251 and LN229 cells $\left(1 \times 10^{4}\right.$ cells/well $)$ in $150 \mu$ of serum-free medium were added into the upper compartment, while $500 \mu \mathrm{l}$ medium with $10 \%$ serum was added into the lower chamber. After culturing at $37^{\circ} \mathrm{C}$ for $24 \mathrm{~h}$, the trans-membrane cells were fixed with methanol and stained with crystal violet. Then, the number of migrated or invasive cells was counted with a microscope (Nikon) with an enlargement factor of $100 \times$.

Wound healing assay. Cell migration ability was also analyzed by the wound healing assay. Transfected U251 and LN229 cells were cultivated in 6-well plates for $24 \mathrm{~h}$ in DMEM containing 5\% FBS. Then a wound was made with the tip of a sterile pipette $(200 \mu \mathrm{l})$ in the monolayer cells. Afterward, cells were gently washed with phosphatebuffered saline (PBS; Beyotime, Shanghai, China) twice and maintained in a serum-free medium for $24 \mathrm{~h}$. To ensure true detection of migration, the cells were exposed to a DNA synthesis inhibitor mitomycin C $(10 \mu \mathrm{g} / \mathrm{ml}$; Abcam, Cambridge, MA, USA) for $2 \mathrm{~h}$ before wound healing. Finally, the wound width was respectively photographed at $0 \mathrm{~h}$ and $24 \mathrm{~h}$ under a microscope (Nikon) with $40 \times$ magnification.
Western blot analysis. For protein analysis, the proteins were isolated from tissues or cultured cells with RIPA lysis buffer (Beyotime). Protein samples were subjected to electrophoresis on $10 \%$ sodium dodecyl sulfate-polyacrylamide gel (SDS-PAGE) and then transferred to nitrocellulose membranes (Solarbio). Next, the membranes were soaked in $5 \%$ nonfat milk and incubated with primary antibodies at $4{ }^{\circ} \mathrm{C}$ overnight. The primary antibodies were shown as follows: anti-proliferating cell nuclear antigen (PCNA) (ab152112; 1:1000 dilution; Abcam), cyclinD1 (ab226977; 1:2000 dilution; Abcam), anti-cleaved-caspase 3 (ab2302; 1:1000 dilution; Abcam), anti-matrix metalloprotein 2 (MMP-2; ab97779; 1:2000 dilution; Abcam), anti-MMP-9 (ab38898; 1:1000 dilution; Abcam), anti-RAB21 (ab88762; 1:1000 dilution; Abcam), anti-GAPDH (ab181602; 1:5000 dilution; Abcam). GAPDH was used as an inner reference. Then, the proteins were incubated with the secondary antibodies against goat anti-mouse or anti-rabbit horseradish peroxidase (HRP)-conjugated IgG antibody (ab6728 or ab6721, 1: 2000 dilution, Abcam). Finally, the immunoreactive bands were visualized using an enhanced chemiluminescent (ECL) kit (Solarbio), and the density of protein signaling was assessed by the Quantity One software (Bio-Rad, Hercules, CA, USA).

Xenograft model. BALB/c nude mice (male, 4-week-old) were bought from Beijing Vital River Laboratory Animal Technology (Beijing, China) and randomly divided into 2 groups ( $n=5 /$ group). U251 cells $\left(2 \times 10^{6}\right)$ were stably transfected with lentiviral vectors of sh-hsa_circ_0030018 or sh-NC using Lipofectamine 3000, and then subcutaneously injected into the left flank of nude mice. Tumor volume was calculated by measuring the formed tumor size every 5 days with the formula: $0.5 \times$ length $\times$ width $^{2} .25$ days later after inoculation, the mice were euthanized by the cervical dislocation to remove the tumors for weight and further molecular analyses. The current animal assays had procured the permission of the Animal Ethics Committee of Jiaozhou People's Hospital.

Dual-luciferase reporter assay. The molecular targets of circ_0030018 or miR-1297 were predicted via online bioinformatics database starBase (http://starbase.sysu.edu. $\mathrm{cn} /$ ). The wild-type luciferase reporter plasmids (wt-hsa circ_0030018 and wt-RAB21 3'UTR) and their mutants (mut-hsa_circ_0030018 and mut-RAB21 3'UTR) were generated through inserting the wild-type sequence of hsa circ_0030018 or RAB21 3'UTR harboring miR-1297 complementary sites or mutant-type sequence of hsa_circ_0030018 or RAB21 3'UTR into pGL3 promoter vectors (Invitrogen), respectively. The constructed reporter vectors were co-transfected with miR-1297 mimics or NC mimics into U251 and LN229 cells. After $48 \mathrm{~h}$ transfection, the relative luciferase activity was detected with a Dual-Lucy Assay Kit (Solarbio).

RNA immunoprecipitation (RIP) assay. For the verification of the relationship between hsa_circ_0030018 or RAB21 and miR-1297, Magna RIP ${ }^{\mathrm{TM}}$ RNA-Binding Protein 
Immunoprecipitation kit (Millipore, Billerica, MA, USA) was utilized to execute the RIP assay. U251 and LN229 cells were lysed in RIP lysis buffer (Sigma-Aldrich, St. Louis, MO, USA). The cell lysate was incubated with antibodies against immunoglobulin G (anti-IgG, Abcam) or antibodies against argonaute 2 (anti-Ago2, Abcam) conjugated with magnetic beads (Sigma-Aldrich) at $4{ }^{\circ} \mathrm{C}$ overnight. Afterward, proteinase K (Sigma-Aldrich) was used to digest the protein in magnetic beads. Then, qRT-PCR was used to measure the enrichment of hsa_circ_0030018, miR-1297, and RAB21 in the anti-Ago2 or anti-IgG group.

RNA pull-down assay. The biotin-coupled miR-1297 probe (Bio-miR-1297) and control oligo probe (Bio-NC) were synthesized by RiboBio (Guangzhou, China). Briefly, U251 and LN229 cells were lysed using a lysis buffer. Bio-miR-1297 or Bio-NC was incubated with C-1 magnetic beads (Life Technologies, Carlsbad, CA, USA) to generate the probe-coated beads. Thereafter, the probe-coated beads were incubated with sonicated U251 and LN229 cells. Finally, the RNA complexes were extracted with the RNeasy Mini Kit (Qiagen). The levels of hsa_circ_0030018 and RAB21 were assessed via qRT-PCR.

Statistical analysis. The results were presented as mean \pm standard deviation obtained from 3 times repeated independent experiments. GraphPad Prism 6.0 software (GraphPad Software Inc., La Jolla, CA, USA) was utilized for data statistical analysis. Statistical significance of data between 2 groups or among multiple groups was assessed by Student's t-test or one-way analysis of variance followed by Tukey post-hoc test in specific circumstances, respectively. A p-value $<0.05$ indicated a significant difference. Furthermore, the linear correlation analysis among hsa_circ_0030018, miR-1297, and RAB21 was performed using Pearson's correlation coefficient analysis.

\section{Results}

hsa_circ_0030018 was upregulated in glioma cells. First, the expression of hsa_circ_0030018 in glioma cell lines (A172, U251, LN229, and T98) and normal cell line NHAs was detected by qRT-PCR. The results revealed that hsa circ_0030018 was highly expressed in glioma cells relative to NHAs, especially in U251 and LN229 cells (Figure 1A). Therefore, U251 and LN229 cells were utilized to further explore the role of hsa_circ_0030018 in glioma cells. To confirm the circular characterization of hsa_circ_0030018, the expression of hsa_circ_0030018 and linear POSTN mRNA was probed in U251 and LN229 cells after RNAse $\mathrm{R}$ or transcriptional inhibitor Actinomycin $\mathrm{D}$ treatment. The results indicated that RNAse $\mathrm{R}$ could degrade linear RNA (POSTN) rather than circRNA (hsa_circ_0030018) (Figures 1B, 1C). Furthermore, Actinomycin D treatment revealed that hsa_circ_0030018 was more stable than linear POSTN mRNA (Figures 1D, 1E). Therefore, these data confirmed that hsa_circ_0030018 was a circular RNA and might play a pivotal role in glioma progression.

Silenced hsa_circ_0030018 restrained the proliferation, metastasis, and induced cell apoptosis and cycle arrest in glioma. For exploring the role of hsa_circ_0030018 in glioma, hsa_circ_0030018 expression was knocked down in
A

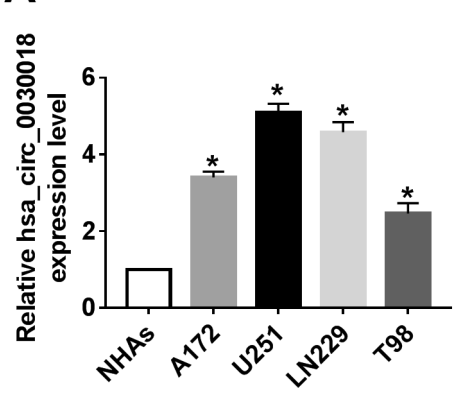

D

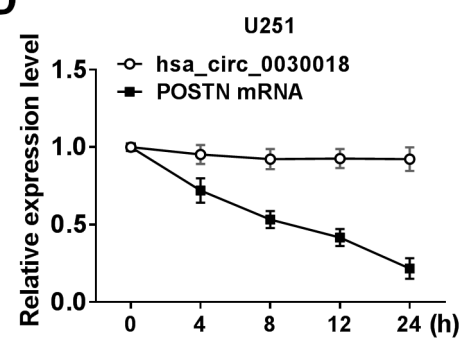

B
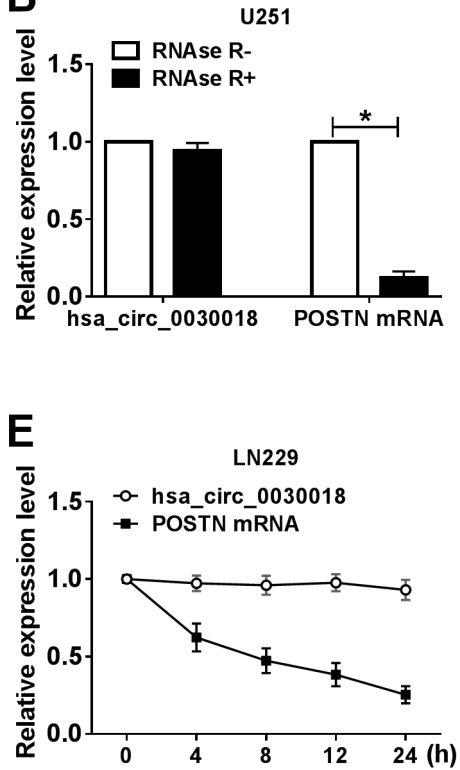

C

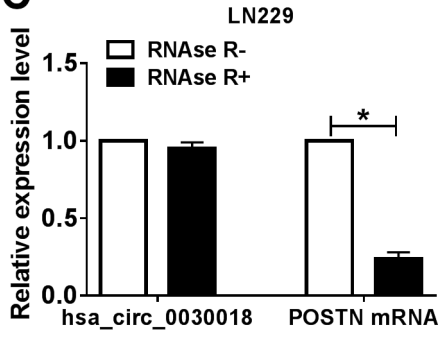

Figure 1. hsa_circ_0030018 was upregulated in glioma cells. A) The level of hsa_circ_0030018 in NHAs, A172, U251, LN229, and T98 cells was measured by qRT-PCR. B, C) hsa_circ_0030018 and linear POSTN levels were detected by qRT-PCR in U251 and LN229 cells exposed to RNAse R. D, E) The levels of hsa_circ_0030018 and POSTN were examined by qRT-PCR after Actinomycin D stimulation. ${ }^{*}$ p $<0.05$ 
U251 and LN229 cells. The results indicated that the expression of hsa_circ_0030018 was distinctly declined after hsa circ_0030018 silence (si-hsa_circ_0030018\#1, \#2 and \#3), while the expression of POSTN mRNA had no significant changes (Figures 2A, 2B). Meanwhile, si-hsa_circ_0030018\#1 with the highest transfection efficiency was used for further experiments. Subsequently, the biological functions of hsa_ circ_0030018 in glioma were evaluated. MTT and colony formation assays showed that hsa_circ_0030018 remarkably suppressed the viability and colony formation ability of U251 and LN229 cells (Figures 2C-2E). Moreover, flow cytometry results indicated that silenced hsa_circ_0030018 enhanced cell apoptosis and cell cycle distribution in the G1 phase (Figures 2F, 2G). Besides, the interference of hsa_ circ_0030018 suppressed migration and invasion of U251 and LN229 cells, which was demonstrated by the Transwell assay (Figures 2H, 2I). Conformably, the wound healing assay also manifested that the wound closure rate was lessened by the hsa_circ_0030018 knockdown in U251 and LN229 cells (Supplementary Figure S1), indicating that the hsa circ_0003732 silence impeded cell migration. In addition, the protein expression levels of viability-related marker (PCNA), cycle-related marker (CyclinD1), and metastasisrelated markers (MMP-2 and MMP-9) were dramatically restrained by silenced hsa_circ_0030018, while apoptosisrelated marker (cleaved-caspase 3) was enhanced, which once again confirmed the above results (Figures 2J-2L). These results suggested that the hsa_circ_0030018 knockdown could repress glioma progression in vitro.

At the same time, a mice xenograft model was also used to explore the effect of circ_0030018 silence on glioma tumor growth in vivo. Therein, U251 cells with stable transfection of sh-hsa_circ_0030018 or sh-NC were subcutaneously injected into nude mice to induce a xenograft model. Through monitoring the tumor size every 5 days after the first injection on day 5 , we found that the tumor volume was significantly decreased after the circ_0030018 knockdown (Figure 2M). Moreover, after 25 days, the tumor weight was remarkably reduced in the sh-hsa_circ_0030018 group in comparison to the sh-NC group (Figure $2 \mathrm{~N}$ ). Additionally, the level of circ_0030018 was markedly decreased in the sh-hsa_circ_0030018 group (Figure 2O). These findings indicated the tumor-promoting role of circ_0030018 in glioma in vivo.

hsa_circ_0030018 was a sponge for miR-1297. Subcellular fractionation assay revealed that hsa_circ_0030018 was principally located in the cytoplasm (Figure 3A). Using the starBase, we found that hsa_circ_0030018 had complementary binding sites with miR-1297 (Figure 3B). As depicted in Figure 3C, miR-1297 was lowly expressed in glioma cell lines rather than normal NHAs cell line. To confirm the interaction between hsa_circ_0030018 and miR-1297, the dualluciferase reporter assay, RIP assay, and RNA-pull down assay were conducted. Dual-luciferase reporter assay revealed that the miR-1297 overexpression significantly reduced the luciferase activity in the wt-hsa_circ_0030018 group, while it had no effect on the luciferase activity in the mut-hsa circ_0030018 group in U251 and LN229 cells (Figures 3D, 3E), implying the interaction between hsa_circ_0030018 and miR-1297. Furthermore, the RIP assay indicated that the levels of hsa_circ_0030018 and miR-1297 were visibly enriched in the anti-Ago2 group compared with that in the anti-IgG group (Figures 3F, 3G), and the RNA-pull down assay suggested that the enrichment of hsa_circ_0030018 was increased in the Bio-miR-1297 group relative to the Bio-NC group (Figure $3 \mathrm{H}$ ). What's more, the expression of miR-1297 was distinctly elevated in U251 and LN229 cells after hsa_circ_0030018 interference and depressed after hsa_ circ_0030018 overexpression (Figures 3I, 3J). These above results testified that hsa_circ_0030018 could directly interact with miR-1297 in glioma cells.

Interference of miR-1297 could reverse the inhibiting effect of hsa_circ_0030018 knockdown on glioma progression. To probe whether miR-1297 was involved in hsa_circ_0030018-mediated glioma development, U251 and LN229 cells were transfected with si-NC, si-hsa circ_0030018\#1, si-hsa_circ_0030018\#1 + NC inhibitor, or si-hsa_circ_0030018\#1 + miR-1297 inhibitor. The qRT-PCR results suggested that miR-1297 inhibitor could invert the promotion effect of si-hsa_circ_0030018 silence on miR-1297 expression, implying that the successful transfection of si-hsa_circ_0030018\#1 and miR-1297 inhibitor (Figure 4A). Simultaneously, interference of miR-1297 attenuated the suppressive effects of hsa_circ_0030018 silence on cell proliferation, colony formation, migration, invasion, and enhancement effects on apoptosis and cell cycle arrest in the G1 phase (Figures 4B-4I). In addition, miR-1297 inhibitor also overturned the decreasing effect of si-hsa_circ_0030018\#1 on the protein levels of PCNA, CyclinD1, MMP-2, and MMP-9, as well as the enhancive effect on the protein level of cleaved-caspase 3 in U251 and LN229 cells (Figures 4J-4O). Taken together, it was concluded that the knockdown of hsa circ_0030018 could inhibit glioma progression via increasing miR-1297 expression.

miR-1297 directly targeted RAB21 in glioma. RAB21 was predicted to be a candidate target of miR-1297 by starBase and the complementary sequence between them appeared in Figure 5A. Interestingly, contrary to the declined expression of miR-1297 in glioma cells, the mRNA and protein levels of RAB21 were overtly raised (Figures 5B, 5C). Then, we constructed wt-RAB21 3'UTR and mut-RAB21 3'UTR to confirm the correlation between miR-1297 and RAB21. The results showed that the luciferase activity of wt-RAB21 3'UTR reporter vector was clearly reduced in U251 and LN229 cells after the hmiR-1297 overexpression, while there was no remarkable change in that of the mut-RAB21 3'UTR group (Figures 5D, 5E). RIP and RNA-pull down assays also verified the interaction between miR-1297 and RAB21 (Figures 5F-5H). More interestingly, the mRNA and protein abundances of RAB21 were both decreased in U251 and 

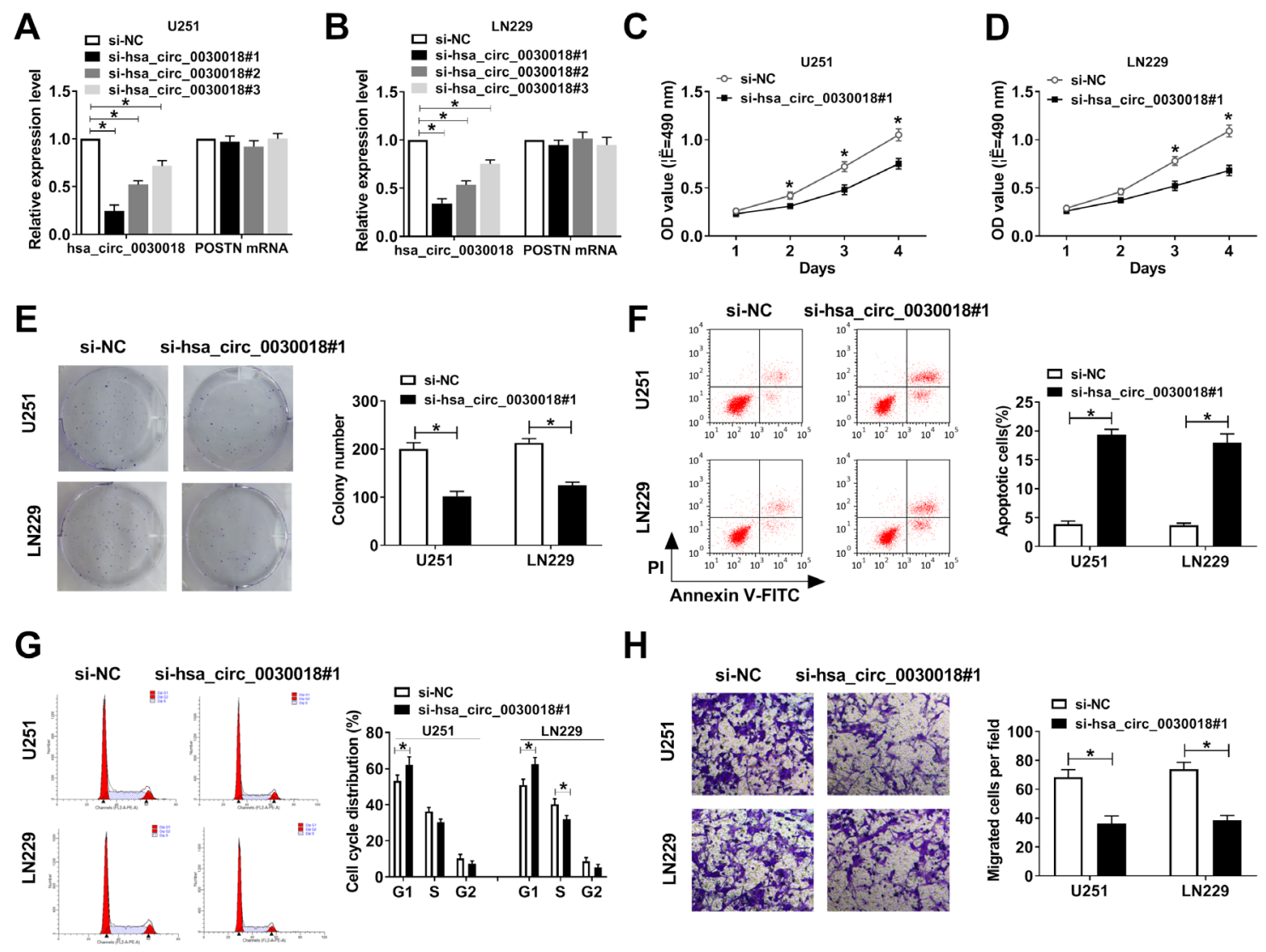

H

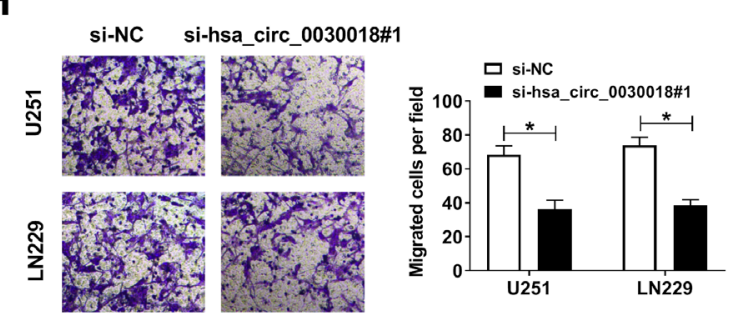

I
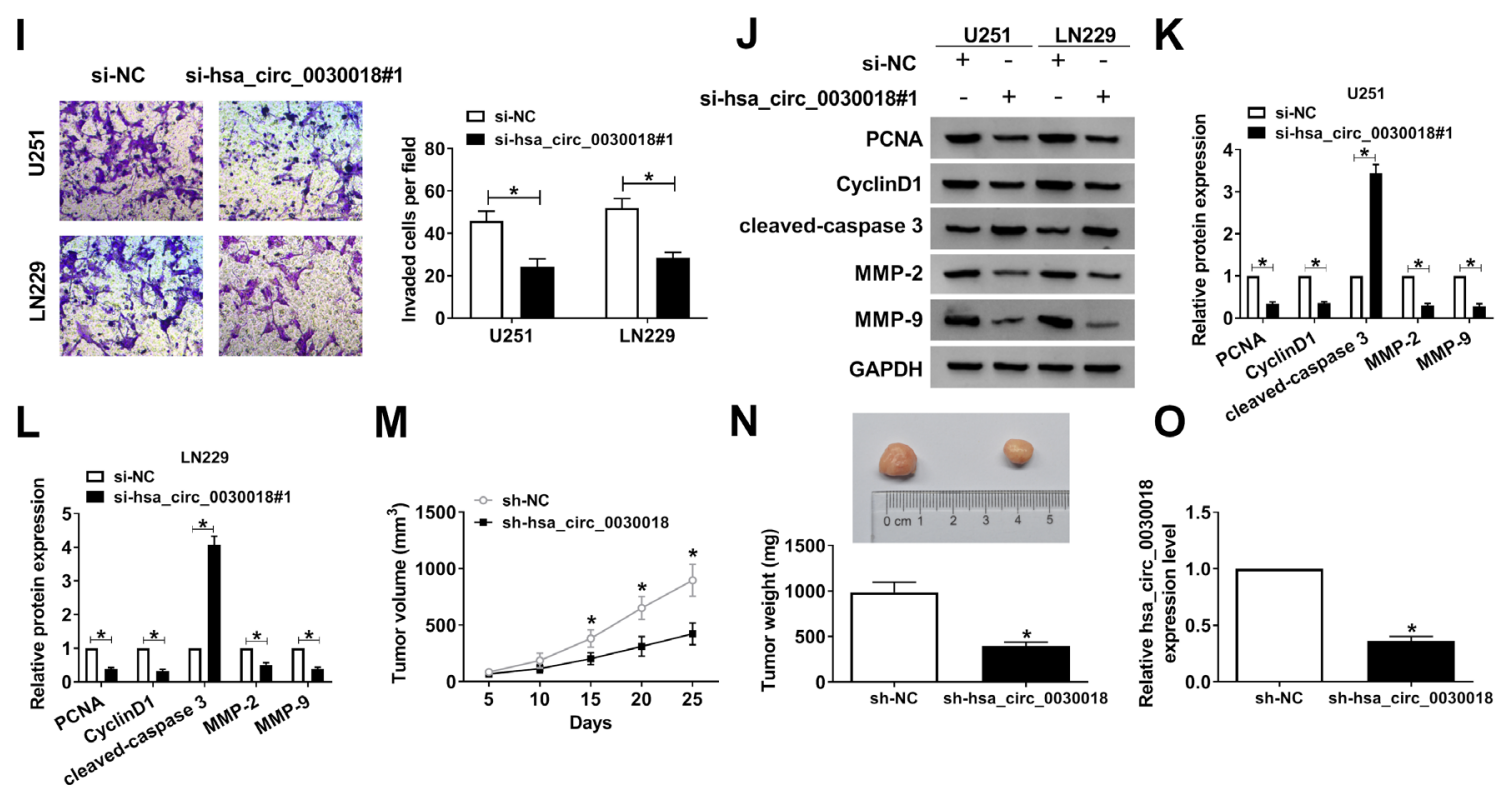

Figure 2. The influence of hsa_circ_0030018 knockdown on glioma development. A, B) The knockdown efficiency of hsa_circ_0030018 (si-hsa_ circ_0030018\#1, \#2, and \#3) in U251 and LN229 cells was detected using qRT-PCR. (C-L) U251 and LN229 cells were transfected with si-NC or si-hsa circ_0030018\#1. C, D) Cell viability was detected in cells after transfection for 1 day, 2 days, 3 days, or 4 days using MTT assay. E) The colony formation was analyzed in cells by colony formation assay. F, G) The apoptotic rate and cell cycle distribution were measured by flow cytometry. The apoptotic rate represented the ratio of cells in right lower and upper quadrants. $\mathrm{H}, \mathrm{I})$ Cell migration and invasion were tested via Transwell assay (100 $\times$ ). J-L) The protein abundances of PCNA, CyclinD1, cleaved-caspase 3, MMP-2, and MMP-9 in cells were examined using western blot. M-O) U251 cells transfected with sh-hsa_circ_0030018 or sh-NC were subcutaneously injected into nude mice to induce a xenograft model. (M) Tumor volume was measured every 5 days after 5 days of injection through a caliper. (N) After 25 days, mice were sacrificed and the resected tumors were weighed. (O) The level of hsa_circ_0030018 was examined using qRT-PCR. ${ }^{*}$ p $<0.05$ 
A

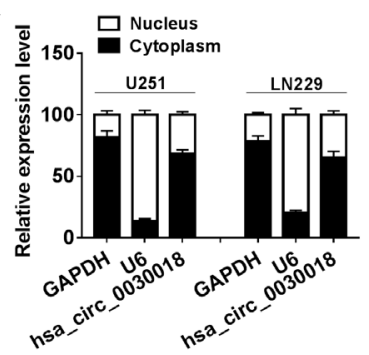

B

wt-hsa_circ_0030018

hsa-miR-1297

mut-hsa_circ_0030018

\section{5' UUCCUCAGCCUACUUGAA 3' \\ | | । I | | | | | \\ 3' GUGGACUU--AAUGAACUU 5' \\ 5' UUGACCAGCCAUAGCUCA 3'}

C

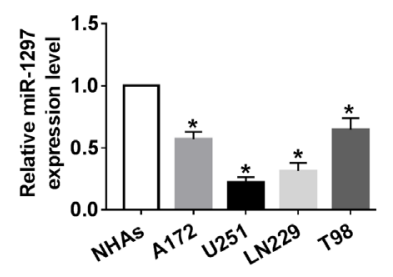

D

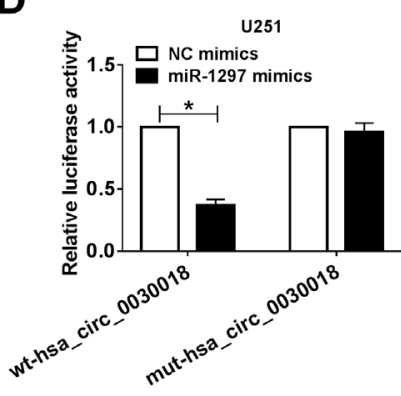

H

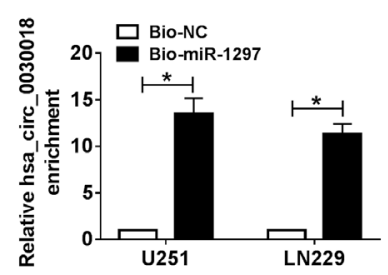

E

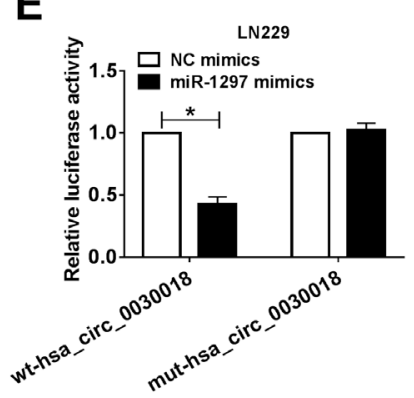

I

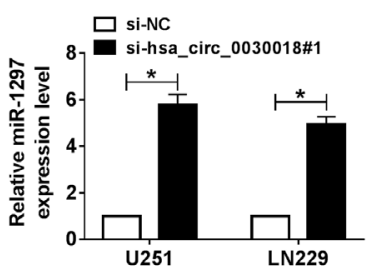

$\mathbf{F}$

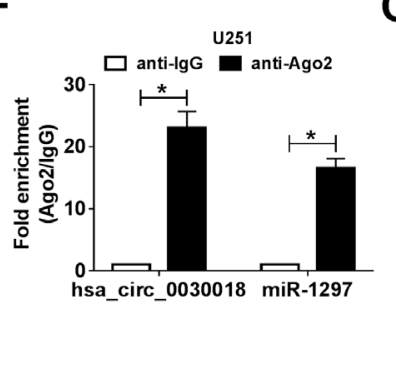

J

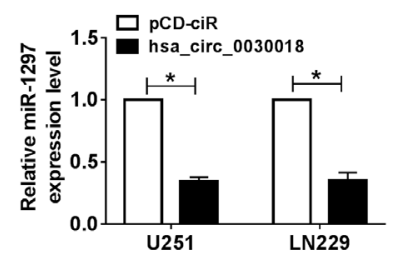

G

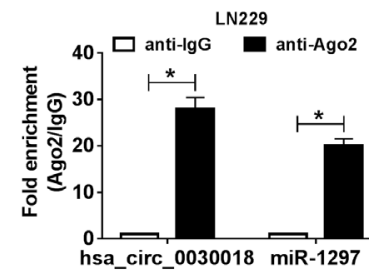

Figure 3. hsa_circ_0030018 directly targeted miR-1297. A) Subcellular fractionation assay was conducted to detect the levels of hsa_circ_0030018 in the cytoplasm and the nucleus of U251 and LN229 cells. B) The predicted binding sequence between hsa_circ_0030018 and miR-1297 was exhibited. C) miR-1297 abundance was examined in glioma cells using qRT-PCR. D, E) Luciferase activity was detected in U251 and LN229 cells co-transfected with wt-hsa_circ_0030018 or mut-hsa_circ_0030018 and miR-1297 mimics or NC mimics. F, G) RIP assay was carried for the assessment of hsa circ_0030018 and miR-1297 levels in the anti-Ago2 or the anti-IgG group. H) The enrichment of hsa_circ_0030018 was analyzed via the RNA-pull down assay in the Bio-miR-1297 or the Bio-NC group. I, J) The miR-1297 level was tested in U251 and LN229 cells with transfection of si-NC, sihsa_circ_0030018\#1, pCD-ciR, or hsa_circ_0030018. ${ }^{*}$ p $<0.05$

LN229 cells after miR-1297 overexpression, while increased by a miR-1297 inhibitor (Figures 5I-5L). Thus, all these findings affirmed that RAB21 was a target of miR-1297 in glioma.

Overexpression of RAB21 counteracted the blocking effects of miR-1297 on glioma cells progression. In order to investigate the function of miR-1297 in glioma and whether it required RAB21, U251 and LN229 cells were co-transfected with miR-1297 mimics, miR-1297 mimics + RAB21, or corresponding negative controls. The transfection of the RAB21 overexpression vector effectively restored the abundance of RAB21 in U251 and LN229 cells, which was declined by miR-1297 mimics transfection (Figures $6 \mathrm{~A}, 6 \mathrm{~B})$. In addition, miR-1297 overexpression obviously restrained cell proliferation, colony formation, migration, invasion, and reinforced apoptosis and cell cycle arrest in the G1 phase (Figures 6C-6P). Nevertheless, RAB21 addition partly overturned the effect of miR-1297 on these events (Figures 6C-6P) in U251 and LN229 cells. Hence, we concluded that miR-1297 could regulate glioma progression by targeting RAB21.

hsa_circ_0030018 regulated RAB21 expression by sponging miR-1297. To perfect the hypothesis of the hsa circ_0030018/miR-1297/RAB21 regulatory network, we measured the influence of hsa_circ_0030018 on RAB21 expression. U251 and LN229 cells were transfected with si-NC, si-hsa_circ_0030018\#1, si-hsa_circ_0030018\#1 + NC inhibitor, or si-hsa_circ_0030018\#1 + miR-1297 inhibitor. As displayed in Figures $7 \mathrm{~A}$ and $7 \mathrm{~B}$, the mRNA and protein abundances of RAB21 were evidently declined by hsa_circ_0030018 silence, while they were restored by the miR-1297 inhibition. Moreover, hsa_circ_0030018 and RAB21 were upregulated while miR-1297 was downregulated in glioma tissues compared to that in adjacent normal tissues (Figures 7C-7F). Besides, correlation analysis showed that miR-1297 expression was negatively corre- 

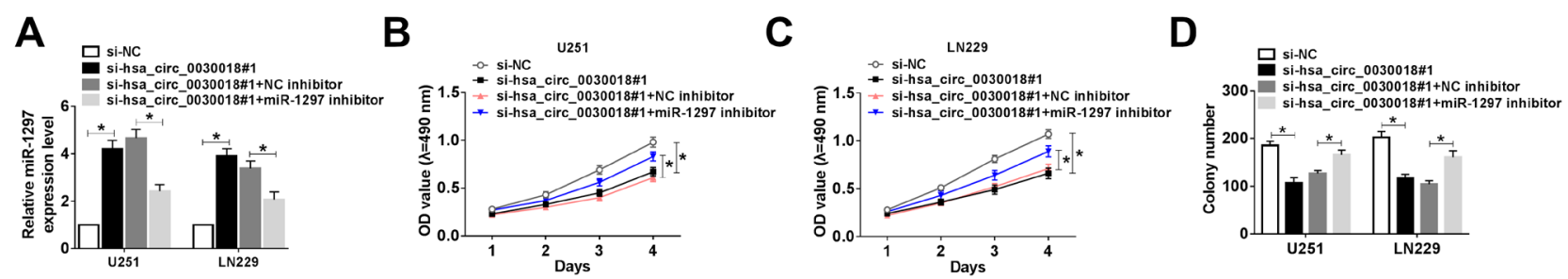

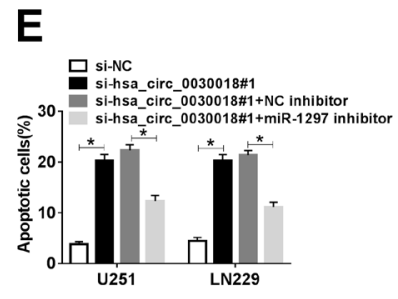

$\mathbf{F}$
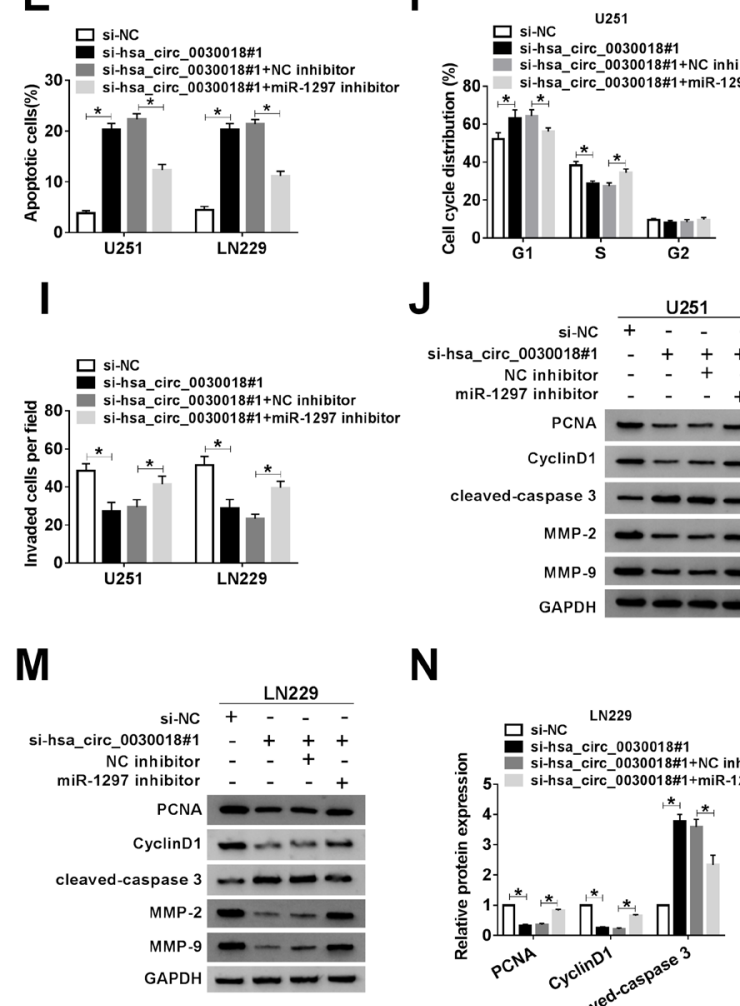

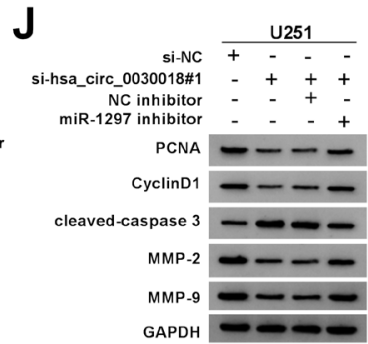

$\mathbf{N}$

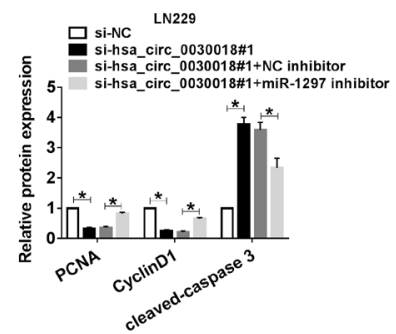

G

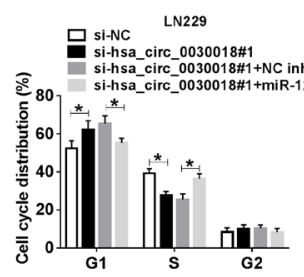

$\mathrm{K}$
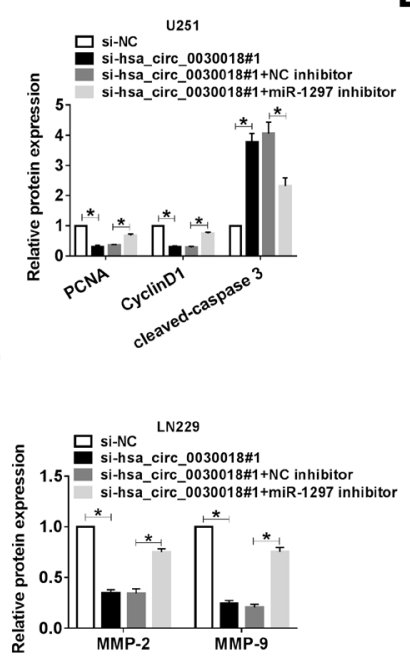

H

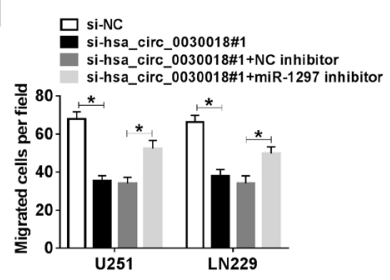

L

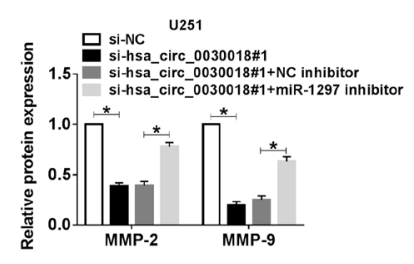

Figure 4. hsa_circ_0030018 regulated glioma progression via modulating miR-1297. U251 and LN229 cells were transfected with si-NC, si-hsa_ circ_0030018\#1, si-hsa_circ_0030018\#1 + NC inhibitor, or si-hsa_circ_0030018\#1 + miR-1297 inhibitor. miR-1297 expression (A), cell viability (B, C), number of colonies (D), apoptotic rate (E), cell cycle distribution (F, G), cell migration and invasion (H, I), and the levels of PCNA, CyclinD1, cleavedcaspase 3, MMP-2, and MMP-9 (J-O) were examined using appropriate methods. ${ }^{\star} \mathrm{p}<0.05$

lated with hsa_circ_0030018 or RAB21 expression, while hsa_circ_0030018 and RAB21 expression were positively correlated (Figures 7G-7I). Collectively, our data confirmed that hsa_circ_0030018 could regulate RAB21 expression via miR-1297 in glioma.

\section{Discussion}

Glioma is the most familiar intracranial tumor, which could cause conspicuous mortality and morbidity [1], so it is indispensable to probe into the novel diagnosis and therapy technology of glioma. The dysregulated circRNAs are involved in glioma development [21]. In this paper, we investigated the role of hsa_circ_0030018 and the corresponding regulatory mechanism of it by regulating the miR-1297/ RAB21 axis in glioma.
Mounting publications have attested the pivotal roles of circRNAs in multifarious human tumors by sponging miRNAs to modulate gene expression [22]. Similarly, the circRNA/miRNA/mRNA axis has also been demonstrated to be concerned with glioma tumorigenesis. For example, circ_0074362/miR-1236-3p/HOXB7 axis and circ-TTBK2/ miR-520b/EZH2 axis contributed to human glioma development $[23,24]$. However, the study on a signal pathway about hsa_circ_0030018 in glioma by targeting miRNAs is exceedingly sparse. To our knowledge, only Yang et al. indicated that hsa_circ_0030018 had a high expression in glioma and it could facilitate cell growth and metastasis via sponging miR-1205 [12]. In keeping with this consequence, this study suggested that hsa_circ_0030018 was upregulated in glioma tissues and cells, and hsa_circ_0030018 deficiency impeded cell proliferation, metastasis, and induced cell 
A

$\begin{array}{lc}\text { wt-RAB21 3'UTR } & \text { 5' UACAUACUUUACUUGAA 3' } \\ \text { hsa-miR-1297 } & \text { 3' GUGGACUUAAUGA | | | | } \\ \text { mut-RAB21 3'UTR } & \text { 5' UACAUACUGGCAACCGA 3' }\end{array}$

B

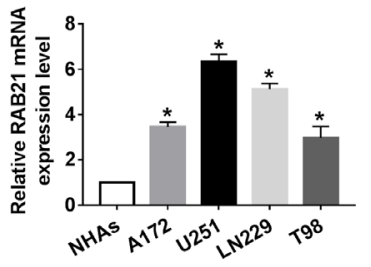

$\mathbf{F}$

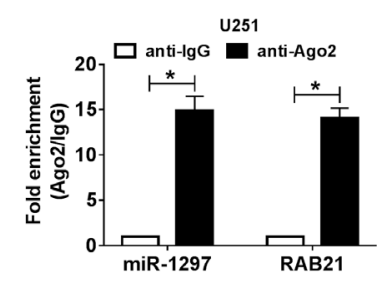

J
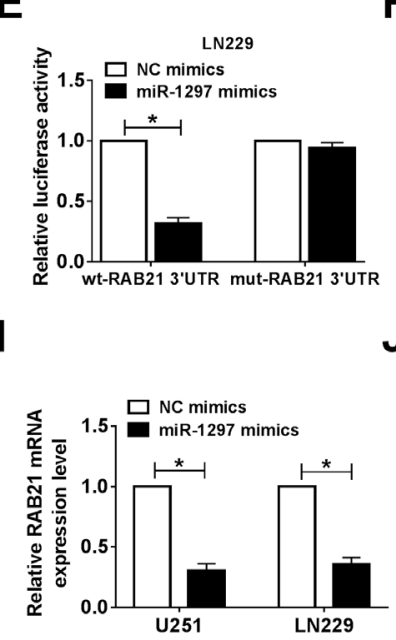

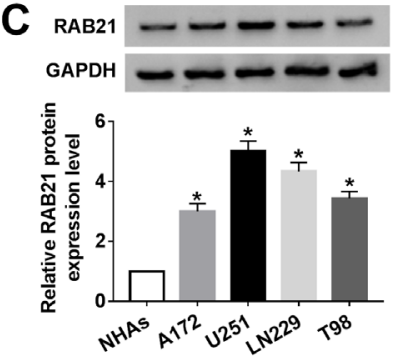

G

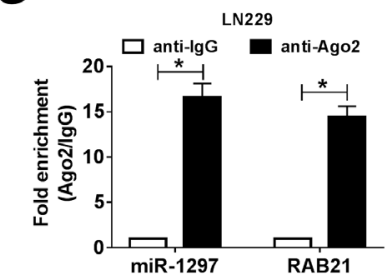

K

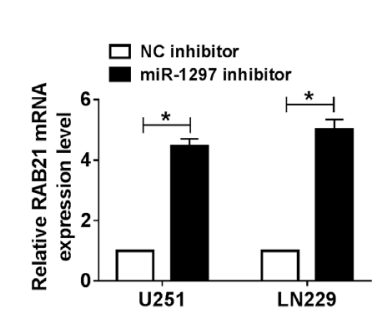

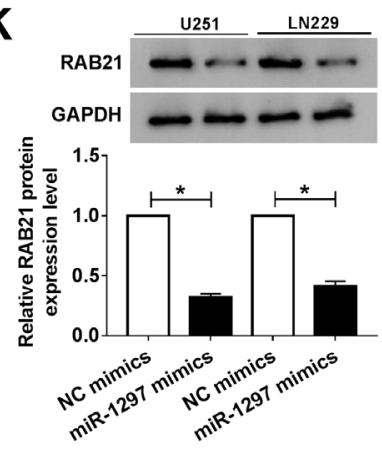

$\mathbf{L}$
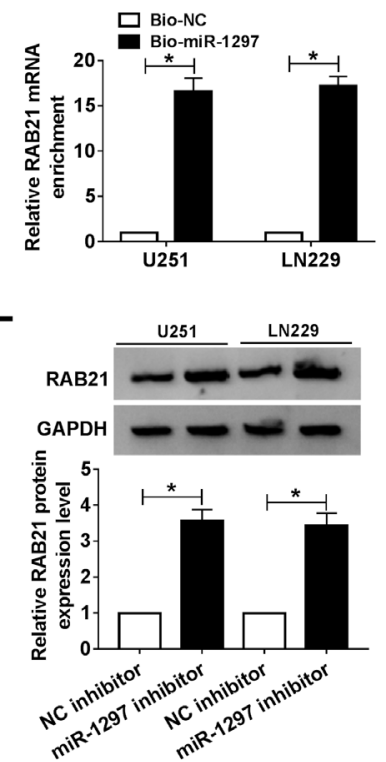

I

H

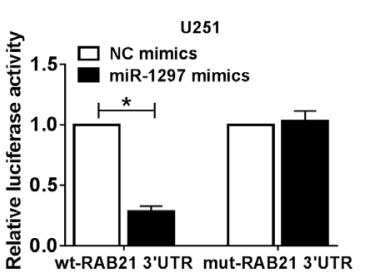

GAPDH $-\infty-$

Figure 5. miR-1297 directly targeted RAB21 in glioma. A) The putative binding sequence of miR-1297 and RAB21. B, C) The mRNA and protein levels of RAB21 were detected in glioma cells via QRT-PCR and western blot. D, E) Luciferase activity was examined in U251 and LN229 cells co-transfected with wt-RAB21 3'UTR and mut-RAB21 3'UTR and miR-1297 mimics or NC mimics. F-H) The interaction between miR-1297 and RAB21 was verified by RIP and RNA-pull down assays in U251 and LN229 cells. I-L) The RAB21 abundance was assessed in U251 and LN229 cells after transfection of miR-1297 mimics, NC mimics, NC inhibitor, or miR-1297 inhibitor using qRT-PCR and western blot. ${ }^{*} \mathbf{p}<0.05$

apoptosis and cycle arrest. Therefore, hsa_circ_0030018 has a latent broad application in the fields of glioma diagnosis and prognosis evaluation. What is more, the expression level of hsa_circ_0030018 in glioma tissues is expected to be a novel biomarker to diagnose glioma. Furthermore, bioinformatic analysis (starBase) predicted that hsa_circ_0030018 had a binding sequence with miR-1297, which was confirmed in a dual-luciferase reporter assay in glioma cells. All these data manifested the oncogenic role of hsa_circ_0030018 in glioma progression, and we speculated it might be relevant to miR-1297.

Accumulating evidence has corroborated that microRNAs serve pivotal roles in glioma genesis and development [25]. miR-1297 has been certified to take part in cancer progression acting as a tumor suppressor or oncogene. For instance, miR-1297 hindered cell growth and metastasis via EphA2 in bladder carcinoma [26], and it impeded tumorigenesis and invasiveness by targeting HMGA2 in hepatocellular 
A

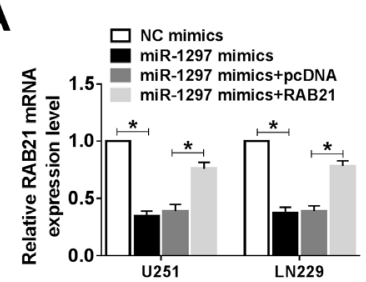

E

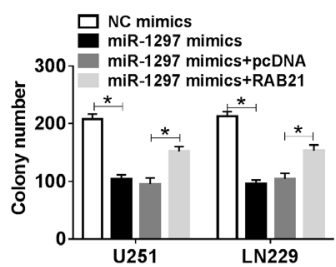

I

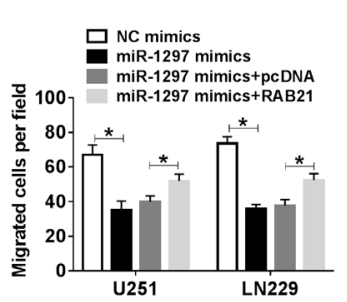

M

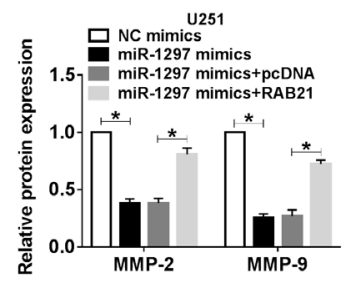

B

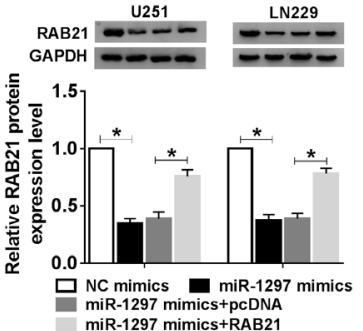

F

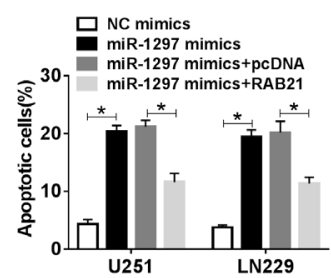

J

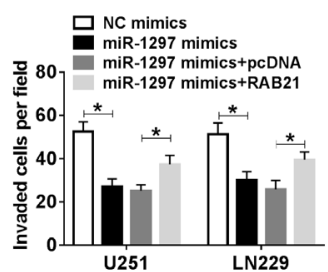

$\mathbf{N}$

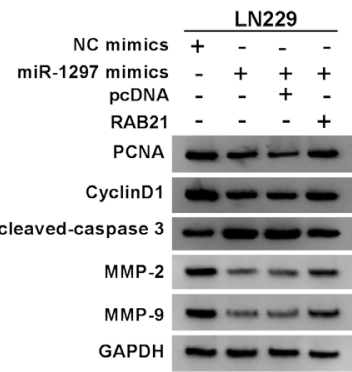

C

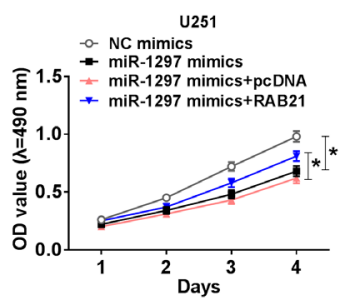

G

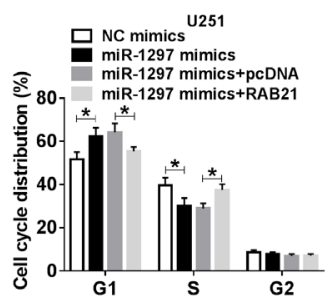

D

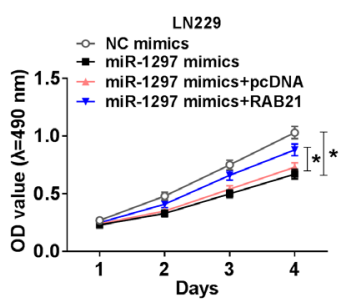

H
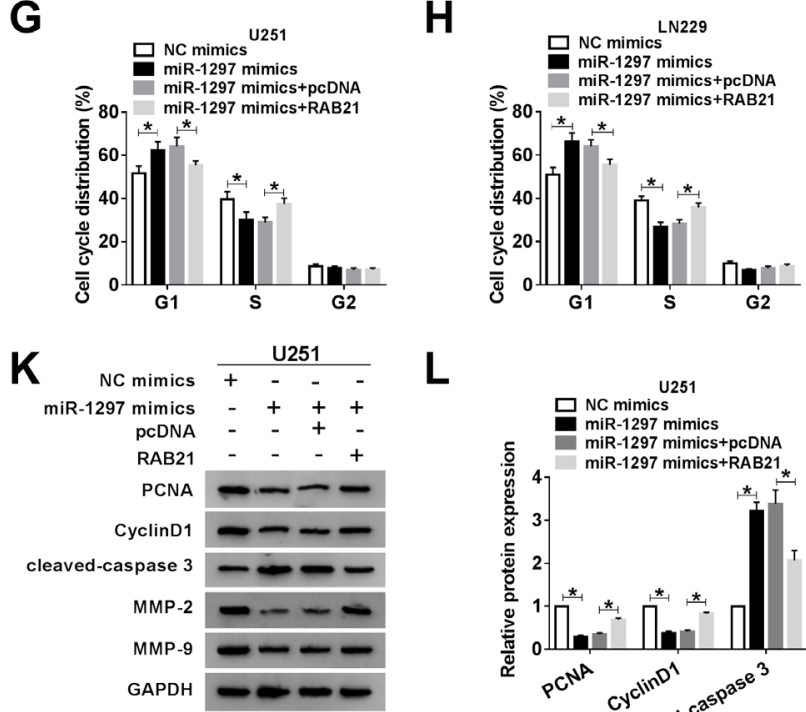

0

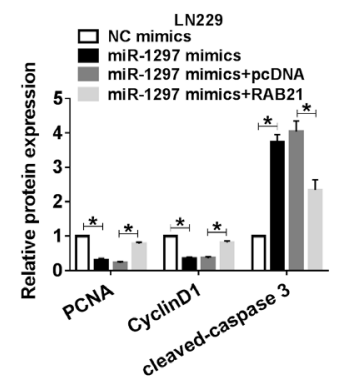

L

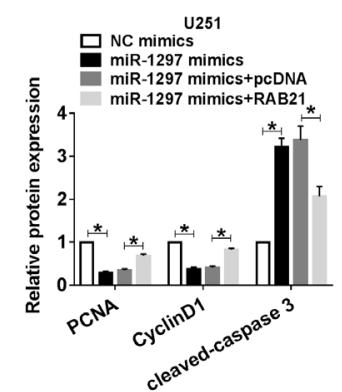

P

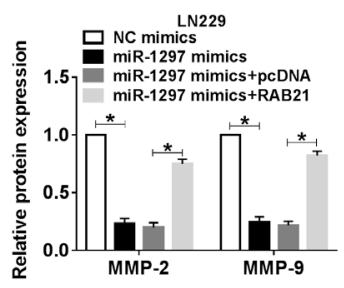

Figure 6. miR-1297 inhibited glioma progression via targeting RAB21. U251 and LN229 cells were transfected with NC mimics, miR-1297 mimics, miR-1297 mimics + pcDNA, or miR-1297 mimics + RAB21. RAB21 mRNA level (A), RAB21 protein level (B), cell viability (C, D), number of colonies (E), apoptotic rate (F), cell cycle distribution (G, H), cell migration and invasion (I, J), and the protein levels of PCNA, CyclinD1, cleaved-caspase 3, MMP-2, and MMP-9 (K-P) were tested using appropriate methods in cells after transfection. ${ }^{\star} \mathbf{p}<0.05$

carcinoma [27]. In the meantime, studies also reported that miR-1297 facilitated the carcinogenic action of liver cancer via targeting RB1 [28], and it also could serve as an oncogenic gene via modulating the PTEN/Akt/Skp2 pathway in NSCLC cells [29]. These antipodal results of miR-1297 in cancers may be attributed to a different type of tumor or complicated tumor microenvironment. However, the researches about the role of miR-1297 in glioma are very rare. Wang et al. reported that miR-1297 level was reduced in glioma and inhibited tumorigenesis [18], and $\mathrm{Li}$ et al. attested that miR-1297 acted as an anti-oncogene in GBM [30]. In accordance with these results, the present study exhibited that the miR-1297 abundance was dramatically reductive in glioma tissues and cells. Moreover, further rescue assays demonstrated that miR-1297 interference partly reversed the suppressive action of hsa_circ_0030018 silence on glioma progression, and that there was a reversed relation between hsa_circ_0030018 and miR-1297 level. The above data showed that miR-1297 as a tumor suppressor and it might be involved in glioma progress by serving as a target of hsa_circ_0030018.

As a member of the Rab subfamily, RAB21 was testified to regulate cell motility and adhesion in several tumor cell types, containing prostate cancer, breast cancer, and liver cancer [31]. RAB21 had a carcinogenic influence in the develop- 

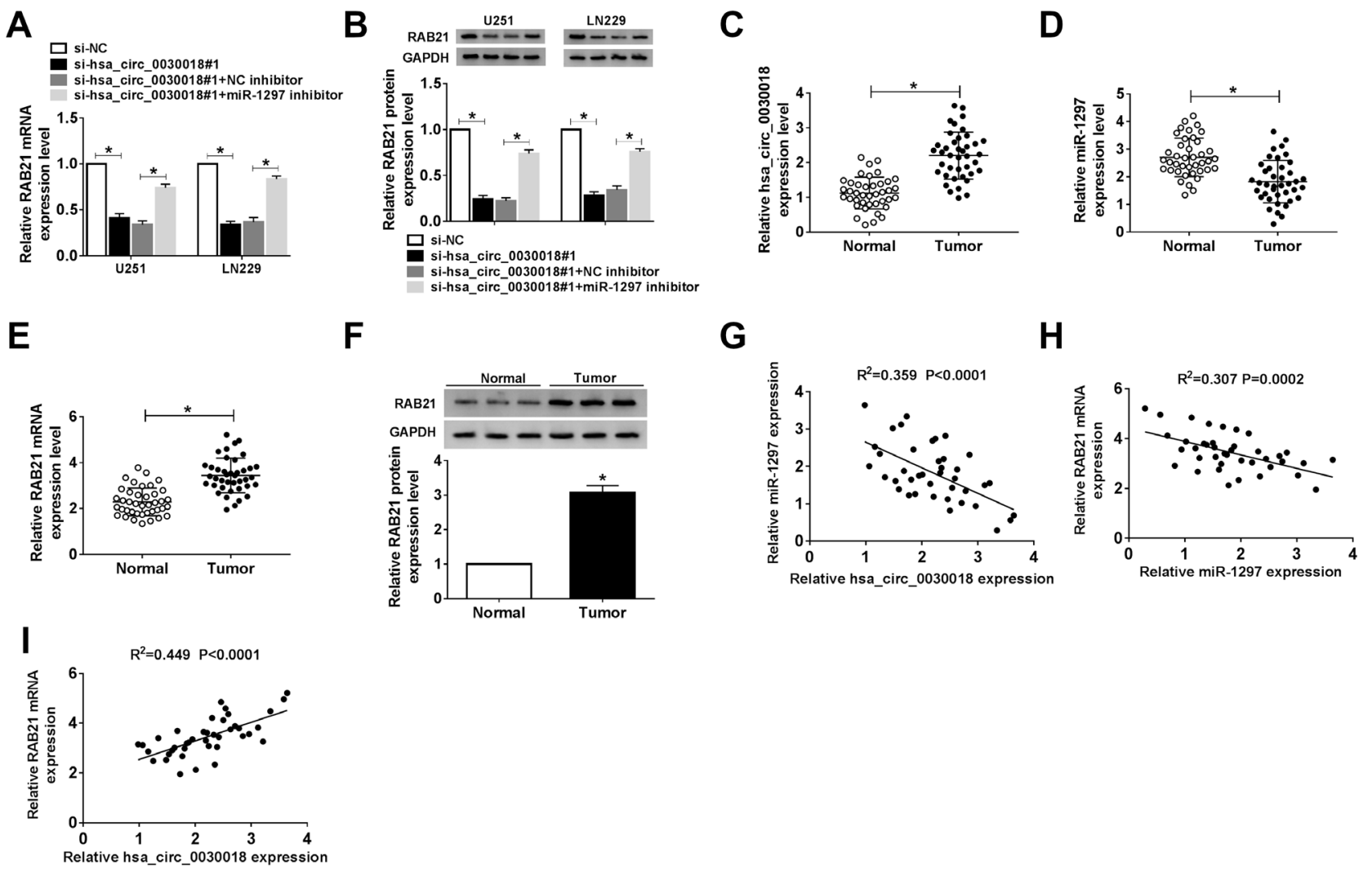

Figure 7. hsa_circ_0030018 regulated RAB21 expression by sponging miR-1297. A, B) RAB21 mRNA and protein abundances were detected in U251 and LN229 cells with transfection of si-NC, si-hsa_circ_0030018\#1, si-hsa_circ_0030018\#1 + NC inhibitor, or si-hsa_circ_0030018\#1 + miR-1297 inhibitor. C, D) The levels of hsa_circ_0030018 and miR-1297 were measured in glioma tissues and normal tissues using qRT-PCR. E, F) RAB21 expression was tested in glioma tissues and normal tissues via qRT-PCR and western blot. G-I) The correlation among hsa_circ_0030018, miR-1297, and RAB21 was assessed using Spearman's correlation analysis. ${ }^{*} \mathbf{p}<0.05$

ment of glioma via accelerating cell proliferation and hinder cell apoptosis [20]. MicroRNAs are virtually involved in the post-transcriptional gene expression by binding to 3'UTR of target genes messenger RNA (mRNA) in cancer [32]. Liu et al. corroborated that miR-200b could restrain glioma genesis by targeting RAB21, RAB23, RAB18, and RAB3B [33]. By using starBase, dual-luciferase reporter assay, RIP assay, and RNA-pull down assay, the current findings indicated that miR-1297 directly targeted RAB21 in glioma, and RAB21 overexpression neutralized the restraining effects of miR-1297 on glioma cells progression. Simultaneously, miR-1297 could negatively regulate RAB21 expression in glioma cells. In addition, the qRT-PCR analysis indicated the upregulation of hsa_circ_0030018 and RAB21, and the downregulation of miR-1297 in glioma tissues. Furthermore, there were significant linear correlations among hsa_circ_0030018, miR-1297, and RAB21 levels. Therefore, it was manifested that hsa circ_0030018 regulated RAB21 expression by sponging miR-1297 in glioma. Collectively, the data gave evidence of the tumorigenic role of hsa_circ_0030018 in glioma development, and this might partly attribute to its regulation of the miR-1297/RAB21 axis. This study provided insights into the participation of hsa_circ_0030018, miR-1297, and RAB21 in glioma tumorigenesis, and offered target candidates for glioma diagnosis and therapeutic strategy.

In conclusion, these findings unveiled that hsa circ_0030018 was overexpressed in glioma, and hsa circ_0030018 interference alleviated the malignant behaviors of glioma in vitro via serving as a miR-1297 sponge to inhibit RAB21. Moreover, the hsa_circ_0030018 depletion impeded tumor growth in vivo. This study for the first time validated the importance of the hsa_circ_0030018/miR-1297/RAB21 axis in glioma, which might provide hopeful targets for glioma treatment.

Supplementary information is available in the online version of the paper.

\section{References}

[1] OSTROM QT, BAUCHET L, DAVIS FG, DELTOUR I, FISHER JL et al. The epidemiology of glioma in adults: a "state of the science" review. Neuro Oncol 2014; 16: 896-913. https://doi.org/10.1093/neuonc/nou087 
[2] CAMELO-PIRAGUA S, KESARI S. Further understanding of the pathology of glioma: implications for the clinic. Expert Rev Neurother 2016; 16: 1055-1065. https://doi.org/10.1080 /14737175.2016.1194755

[3] WEN PY, REARDON DA. Neuro-oncology in 2015: Progress in glioma diagnosis, classification and treatment. Nat Rev Neurol 2016; 12: 69-70. https://doi.org/10.1038/nrneurol.2015.242

[4] REIFENBERGER G, WIRSCHING HG, KNOBBE-THOMSEN CB, WELLER M. Advances in the molecular genetics of gliomas - implications for classification and therapy. Nat Rev Clin Oncol 2017; 14: 434-452. https://doi.org/10.1038/ nrclinonc.2016.204

[5] LIU L, WANG J, KHANABDALI R, KALIONIS B, TAI X et al. Circular RNAs: Isolation, characterization and their potential role in diseases. RNA biology 2017; 14: 1715-1721. https://doi.org/10.1080/15476286.2017.1367886

[6] NG WL, MOHD MOHIDIN TB, SHUKLA K. Functional role of circular RNAs in cancer development and progression. RNA Biol 2018; 15: 995-1005. https://doi.org/10.1080/ 15476286.2018.1486659

[7] ZHANG Y, LIN X, GENG X, SHI L, LI Q et al. Advances in circular RNAs and their role in glioma (Review). Int J Oncol 2020; 57: 67-79. https://doi.org/10.3892/ijo.2020.5049

[8] SUN J, LI B, SHU C, MA Q, WANG J. Functions and clinical significance of circular RNAs in glioma. Mol Cancer 2020; 19: 34. https://doi.org/10.1186/s12943-019-1121-0

[9] XIONG Z, ZHOU C, WANG L, ZHU R, ZHONG L et al. Circular RNA SMO sponges miR-338-3p to promote the growth of glioma by enhancing the expression of SMO. Aging (Albany NY) 2019; 11: 12345-12360. https://doi.org/10.18632/ aging. 102576

[10] DING C, WU Z, YOU H, GE H, ZHENG S et al. CircNFIX promotes progression of glioma through regulating miR378e/RPN2 axis. J Exp Clin Cancer Res 2019; 38: 506. https:// doi.org/10.1186/s13046-019-1483-6

[11] WANG C, TANG D, WANG H, HU G, HU S et al. Circular RNA hsa_circ_0030018 acts as a sponge of miR-599 to aggravate esophageal carcinoma progression by regulating ENAH expression. J Cell Biochem 2019. https://doi.org/10.1002/ jcb. 29507

[12] YANG Y, ZHANG Y, CHEN B, DING L, MU Z et al. Elevation of circular RNA circ-POSTN facilitates cell growth and invasion by sponging miR-1205 in glioma. J Cell Biochem 2019; 120: 16567-16574. https://doi.org/10.1002/jcb.28916

[13] BARTEL DP. MicroRNAs: target recognition and regulatory functions. Cell 2009; 136: 215-233. https://doi.org/10.1016/j. cell.2009.01.002

[14] TRUJILLO RD, YUE SB, TANG Y, O'GORMAN WE, CHEN CZ. The potential functions of primary microRNAs in target recognition and repression. EMBO J 2010; 29: 3272-3285. https://doi.org/10.1038/emboj.2010.208

[15] CHEN Z, MA Y, PAN Y, ZHU H, YU C et al. MiR-1297 suppresses pancreatic cancer cell proliferation and metastasis by targeting MTDH. Mol Cell Probes 2018; 40: 19-26. https:// doi.org/10.1016/j.mcp.2018.06.003
[16] ZHANG X, ZHANG M, GUO Q, HU X, ZHAO Z et al. MicroRNA-1297 inhibits proliferation and promotes apoptosis in gastric cancer cells by downregulating CDC6 expression. Anticancer Drugs 2019; 30: 803-811. https://doi. org/10.1097/CAD.0000000000000776

[17] LIANG X, LI H, FU D, CHONG T, WANG Z et al. MicroRNA-1297 inhibits prostate cancer cell proliferation and invasion by targeting the AEG-1/Wnt signaling pathway. Biochem Biophys Res Commun 2016; 480: 208-214. https://doi. org/10.1016/j.bbrc.2016.10.029

[18] WANG J, XU X, MO S, TIAN Y, WU J et al. Involvement of microRNA-1297, a new regulator of HMGA1, in the regulation of glioma cell growth in vivo and in vitro. Am J Transl Res 2016; 8: 2149-2158.

[19] OPDAM FJ, KAMPS G, CROES H, VAN BOKHOVEN $\mathrm{H}$, GINSEL LA et al. Expression of Rab small GTPases in epithelial Caco-2 cells: Rab21 is an apically located GTPbinding protein in polarised intestinal epithelial cells. Eur J Cell Biol 2000; 79: 308-316. https://doi.org/10.1078/S01719335(04)70034-5

[20] GE J, CHEN Q, LIU B, WANG L, ZHANG S et al. Knockdown of Rab21 inhibits proliferation and induces apoptosis in human glioma cells. Cell Mol Biol Lett 2017; 22: 30. https://doi.org/10.1186/s11658-017-0062-0

[21] HAO Z, HU S, LIU Z, SONG W, ZHAO Y et al. Circular RNAs: Functions and Prospects in Glioma. J Mol Neurosci 2019; 67: 72-81. https://doi.org/10.1007/s12031-018-1211-2

[22] QU S, ZHONG Y, SHANG R, ZHANG X, SONG W et al. The emerging landscape of circular RNA in life processes. RNA Biol 2017; 14: 992-999. https://doi.org/10.1080/15476286.20 16.1220473

[23] DUAN X, LIU D, WANG Y, CHEN Z. Circular RNA hsa_ circ_0074362 Promotes Glioma Cell Proliferation, Migration, and Invasion by Attenuating the Inhibition of miR1236-3p on HOXB7 Expression. DNA Cell Biol 2018; 37: 917-924. https://doi.org/10.1089/dna.2018.4311

[24] YUAN DH, ZHAO J, SHAO GF. Circular RNA TTBK2 promotes the development of human glioma cells via miR-520b/ EZH2 axis. Eur Rev Med Pharmacol Sci 2019; 23: $10886-$ 10898. https://doi.org/10.26355/eurrev_201912_19792

[25] ZHOU Q, LIU J, QUAN J, LIU W, TAN H et al. MicroRNAs as potential biomarkers for the diagnosis of glioma: A systematic review and meta-analysis. Cancer Sci 2018; 109: 2651-2659. https://doi.org/10.1111/cas.13714

[26] PENG G, MENG H, PAN H, WANG W. CircRNA 001418 promoted cell growth and metastasis of bladder carcinoma via EphA2 by miR-1297. Curr Mol Pharmacol 2020. https:// doi.org/10.2174/1874467213666200505093815

[27] LIU Y, LIANG H, JIANG X. MiR-1297 promotes apoptosis and inhibits the proliferation and invasion of hepatocellular carcinoma cells by targeting HMGA2. Int J Mol Med 2015; 36: 1345-1352. https://doi.org/10.3892/ijmm.2015.2341

[28] LIU C, WANG C, WANG J, HUANG H. miR-1297 promotes cell proliferation by inhibiting RB1 in liver cancer. Oncol Lett 2016; 12: 5177-5182. https://doi.org/10.3892/ ol.2016.5326 
[29] BU W, LUO T. miR-1297 Promotes Cell Proliferation of Non-Small Cell Lung Cancer Cells: Involving in PTEN/Akt/ Skp2 Signaling Pathway. DNA Cell Biol 2017; 36: 976-982. https://doi.org/10.1089/dna.2017.3886

[30] LI H, YUAN H. MiR-1297 negatively regulates metabolic reprogramming in glioblastoma via repressing KPNA2. Hum Cell 2020; 33: 619-629. https://doi.org/10.1007/s13577-01900316-7

[31] PELLINEN T, ARJONEN A, VUORILUOTO K, KALLIO $\mathrm{K}$, FRANSEN JA et al. Small GTPase Rab21 regulates cell adhesion and controls endosomal traffic of beta1-integrins. J Cell Biol 2006; 173: 767-780. https://doi.org/10.1083/ jcb.200509019
[32] ALI SYEDA Z, LANGDEN SSS, MUNKHZUL C, LEE M, SONG SJ. Regulatory Mechanism of MicroRNA Expression in Cancer. Int J Mol Sci 2020; 21: https://doi.org/10.3390/ ijms 21051723

[33] LIU Q, TANG H, LIU X, LIAO Y, LI H et al. miR-200b as a prognostic factor targets multiple members of RAB family in glioma. Med Oncol 2014; 31: 859. https://doi.org/10.1007/ s12032-014-0859-x 
https://doi.org/10.4149/neo_2020_200702N682

\section{CircRNA hsa_circ_0030018 regulates the development of glioma via regulating the miR-1297/RAB21 axis}

Dewen SONG, Lin YE, Zengliang XU, Yanfei JIN, Lintao ZHANG*

\section{Supplementary Information}
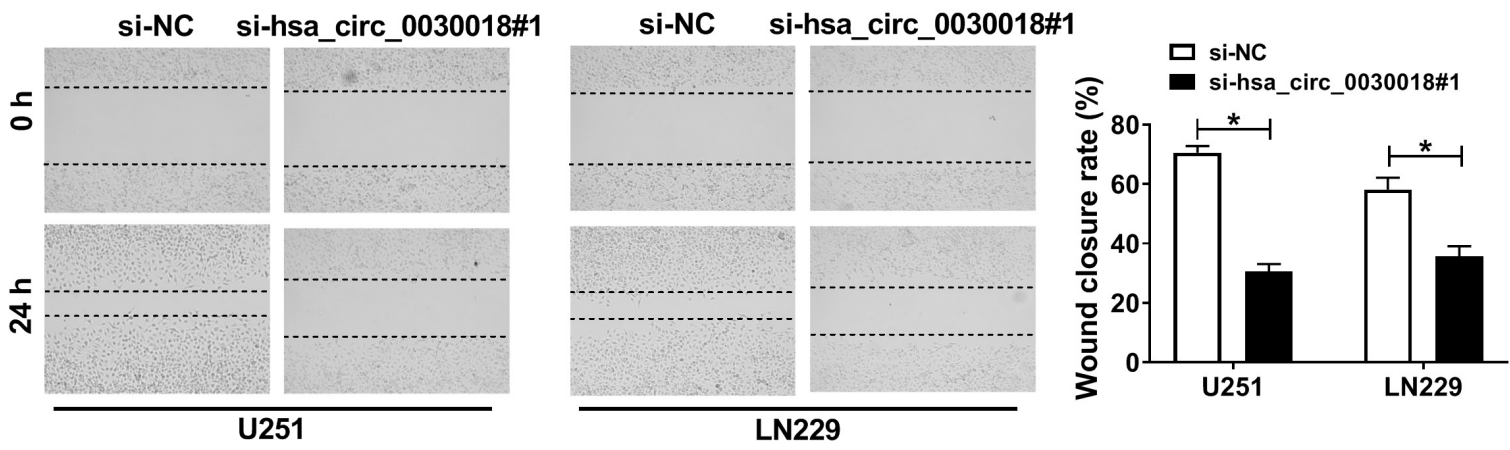

Supplementary Figure S1. Hsa_circ_0030018 knockdown inhibited cell migration in glioma cells. The wound closure rate of U251 and LN229 cells transfected with si-NC or si-hsa_circ_0030018\#1 was tested by wound healing assay. 